\title{
El control judicial como control preventivo de cláusulas abusivas (Corte Suprema)
}

\author{
Comentario de María Elisa Morales Ortiz*
}

Santiago, siete de julio de dos mil dieciséis.

Vistos:

En los autos Rol N ${ }^{\circ}$ 1533-2015 de esta Corte Suprema sobre demanda colectiva por vulneración al interés difuso de los consumidores, deduce recursos de casación en la forma y en el fondo en lo principal y primer otrosí de fojas 445 el Servicio Nacional del Consumidor, en contra de la sentencia de once de noviembre de dos mil catorce, dictada por la Corte de Apelaciones de Santiago, que revocó parcialmente el fallo de primer grado que, a su turno, había acogido parcialmente la demanda, declarando nulas diversas cláusulas, declarando improcedentes las indemnizaciones o reparaciones por no existir un grupo o subgrupo afectado y disponiendo la publicación de dos avisos de acuerdo a lo dispuesto en el artículo 54 de la ley 19.496. La sentencia de la Corte de Apelaciones revocó esta última solo en cuanto por ella no se multó a la denunciada, y en su lugar la condenó a pagar una multa de 50 Unidades Tributarias Mensuales.

Se trajeron los autos en relación.

\section{CONSIDERANDO:}

\section{EN CUANTO AL RECURSO DE CASACIÓN EN LA FORMA}

PRIMERO: Que por el recurso de casación en la forma se denuncia, en primer lugar, la falta de decisión del asunto controvertido en relación con la solicitud de declarar abusivas y nulas las cláusulas "Uso comercial” y "Política de privacidad de Ticketmaster".

SEGUNDO: Que la sentencia de primer grado, confirmada en esta parte por la de alzada, declaró que acogía parcialmente la demanda solo en cuanto declaraba nulas las cláusulas que individualizó en el punto III de su parte resolutiva. En consecuencia, dicha

* Licenciada en Ciencias Jurídicas y Sociales, Universidad Austral de Chile. Profesora de Derecho Civil y Comercial, Departamento de Ciencias Jurídicas, Facultad de Ciencias Jurídicas y Empresariales, Universidad de La Frontera, Temuco, Chile. Correo electrónico: maria.morales@ufrontera.cl. 
sentencia rechazó parcialmente la demanda en relación con las cláusulas no individualizadas en el citado punto, entre las que se encuentran la de "Uso comercial" y la "Política de privacidad de Ticketmaster".

En consecuencia, la sentencia impugnada sí resolvió el asunto controvertido en relación con estas cláusulas, aunque en un sentido contrario del pretendido por la recurrente.

Tercero: Que, en un segundo capítulo, el recurso denuncia que no se decidió el asunto controvertido en relación con la solicitud de imponer multa por infracción del artículo 17 de la ley de protección al consumidor, que impone la obligación de que la letra de los contratos de adhesión no sea inferior a 2,5 milímetros.

CuARto: Que la sentencia estimó que no se había acreditado infracción al artículo 17 de la citada ley. En consecuencia, la sentencia sí decidió el asunto controvertido, en cuanto no accedió a imponer multa por infracción del artículo 17.

Por otra parte, la sentencia impugnada impuso el máximo de la multa genérica que establece su artículo 24, por haber incurrido la denunciada en infracción de los artículos 16 y 43 de la ley de protección al consumidor. La infracción del artículo 17 de esta ley no tiene señalada una multa especial. Por tanto, aún si se los hubiera estimado infringido, la multa no podría haber sido distinta de la que se impuso por infracción de los artículos 16 y 43 de la citada ley, de manera que el vicio denunciado no ocasionaría perjuicio alguno solo reparable con la anulación de la sentencia.

Quinto: Que, en un tercer capítulo, el recurso reclama que la sentencia impugnada no decidió el asunto controvertido en relación con la solicitud de condenar a la demandada al pago de indemnización de perjuicios ocasionados a los consumidores.

Esta denuncia es infundada, pues la sentencia impugnada desestimó expresamente la demanda en este punto, "por no existir grupo o subgrupo afectado".

SeXto: Que en razón de las consideraciones que preceden, el recurso de casación en la forma será desestimado.

\section{EN CUANTO AL RECURSO DE CASACIÓN EN EL FONDO}

SÉptimo: Que por el recurso de casación en el fondo el Servicio Nacional del Consumidor reclama infringido, en primer lugar, el artículo 16 letra g) de la ley 19.496 sobre protección de los derechos de los consumidores. Sostiene que la sentencia impugnada habría incurrido en dicha infracción al confirmar la validez de las cláusulas "Uso Comercial" y "Política de privacidad de Ticketmaster".

Octavo: Que la disposición legal cuya infracción se denuncia es del siguiente tenor:

No producirán efecto alguno en los contratos de adhesión las cláusulas o estipulaciones que... g) En contra de las exigencias de la buena fe, atendiendo para estos efectos 
a parámetros objetivos, causen en perjuicio del consumidor, un desequilibrio importante en los derechos y obligaciones que para las partes se deriven del contrato. Para ello se atenderá a la finalidad del contrato y a las disposiciones especiales o generales que lo rigen. Se presumirá que dichas cláusulas se encuentran ajustadas a exigencias de la buena fe, si los contratos a que pertenecen han sido revisados y autorizados por un órgano administrativo en ejecución de sus facultades legales.

Noveno: Que la cláusula "Uso comercial” dispone:

Ninguno de los anuncios, de este Sitio pueden ser usados por nuestros visitantes dentro de los términos establecidos por Ticektmaster, así como por la legislación de la materia por lo que nos reservamos el derecho a bloquear el acceso a este Sitio o a otros servicios de Ticketmaster, o a cancelar el proceso de adquisición de un boleto o boletos en relación con cualquier persona que se cree ser, o que se cree que está actuando en conexión con cualquier persona que se crea que esté violando la ley o los términos establecidos por los derechos de Ticketmaster, o bien que ha ordenado un número de boletos que excede los límites establecidos. El violar cualquiera de las limitaciones o los términos de este Sitio será considerado como una violación de estos Términos.

La sentencia desestimó la demanda respecto de esta cláusula por estimar que "si bien tiene una redacción poco clara, por si misma no involucra una vulneración manifiesta a la buena fe, sino solo una forma de proteger la utilización de la página web frente a terceros que pudieran obrar en contravención a la legislación vigente”.

La recurrente discrepa de esta consideración, y alega que "el derecho de cancelar unilateralmente el proceso de compra de un boleto por parte del proveedor, frente a una mera sospecha de un acto contrario a la ley, atenta primero contra las exigencias de la buena fe contractual, y segundo, provoca un evidente desequilibrio entre las partes contratantes".

DÉcımo: Que la cláusula transcrita es una de las condiciones aplicables a la venta de entradas para espectáculos a través de un sitio que opera en la red virtual denominada "Internet". Es de la naturaleza de las transacciones a través de esta red que no se produzca un encuentro físico entre dos personas. Esa característica de la red facilita las operaciones fraudulentas. Un tipo de operación fraudulenta es aquella en que una persona realiza una transacción en beneficio propio utilizando, sin autorización, un medio de pago perteneciente a un tercero. Otro tipo de operación fraudulenta es aquella en que una persona realiza una compra masiva de entradas, a objeto de luego revenderlas a un precio mayor. Es cierto, como manifestó la sentencia impugnada, que la redacción de la cláusula "Uso comercial" es oscura. La gramática utilizada sugiere que se trata de una deficiente traducción del inglés. Aún así, es manifiesto que la cláusula tiene por objeto proteger el sitio de Ticketmaster ante operaciones fraudulentas como las mencionadas o análogas. Por otra parte, ante la creencia de que se está haciendo un uso fraudulento del sitio, el operador del mismo tiene que reaccionar inmediatamente. De lo contrario, inevitablemente se producirá un daño a terceros o al público. En efecto, si ante la sospecha de que un comprador está usando un medio de pago de un tercero, 
sin su autorización, el operador del sitio no tiene la facultad de bloquear la operación, el tercero resultará defraudado. Y el operador se exculpará de este fraude alegando que estaba legalmente impedido de bloquear la operación. En consecuencia, la cláusula "Uso comercial" no es contraria a la buena fe, en la medida que otorga al operador del sitio una facultad razonable para protegerse ante su uso fraudulento.

Lo anterior no significa que en ejercicio de la cláusula "Uso comercial" el operador del sitio no pueda cometer errores. Los mecanismos para detectar operaciones fraudulentas pueden ser imperfectos y resultar en el bloqueo de transacciones de buena fe. Pero esto no conlleva la invalidez de la cláusula. Y la validez de la cláusula no impide que quienes en concreto resulten injustamente afectados por ella recurran a la protección que la ley les otorga.

Por estas consideraciones, la sentencia impugnada no ha incurrido en error de derecho al desechar la demanda en relación con la cláusula "Uso comercial".

Undécimo: Que la cláusula "Política de privacidad de Ticketmaster" es del siguiente tenor:

Ticketmaster podrá revelar la información proporcionada por sus Usuarios a terceros, incluyendo patrocinadores, publicistas y/o socios comerciales. Ticketmaster también recolectará información que es derivada de los gustos, preferencias y en general de la utilización que hacen los Usuarios de los Servicios. Dicha información derivada, al igual que la información personal que los Usuarios proporcionen, podrá ser utilizada para diversos objetivos comerciales, como lo es el proporcionar datos estadísticos (por ejemplo: $50 \%$ de nuestros Usuarios son mujeres) a anunciantes potenciales, enviar publicidad a los Usuarios de acuerdo a sus intereses específicos, conducir investigaciones de mercadeo, y otras actividades o promociones que Ticektmaster considere apropiadas. Ticektmaster también podrá revelar información cuando por mandato de ley y/o de autoridad competente le fuere requerido o por considerar de buena fe que dicha revelación es necesaria para: I) cumplir con procesos legales; II) cumplir con el Convenio del Usuario; III) responder reclamaciones que involucren cualquier Contenido que menoscabe derechos de terceros o; IV) proteger los derechos, la propiedad, o la seguridad de Ticketmaster, sus Usuarios y el público en general.

Esta cláusula contiene diversas autorizaciones a Ticketmaster. No son sin embargo autorizaciones que el usuario dé positiva y especialmente. Tampoco son autorizaciones supletorias que el usuario pueda denegar si así lo desea. Son autorizaciones que se entienden concedidas por el consumidor por el solo hecho de usar el sitio. Esta característica resultará determinante para establecer el carácter abusivo de buena parte de estas autorizaciones.

La primera autorización permite a Ticketmaster "revelar la información proporcionada por sus Usuarios a terceros, incluyendo patrocinadores, publicistas y/o socios comerciales”. Esta autorización para revelar información supone los dos procesos previos de obtención y almacenamiento de la información. La obtención de cierta información es ciertamente necesaria para generar las transacciones comerciales de venta de entradas. El operador del sitio necesita, al menos, la identidad del consumidor, la información 
relativa al medio de pago utilizado, e información para el despacho físico o virtual del comprobante de la transacción. Con tal objeto, el operador requerirá cierta información a objeto de proceder a la transacción. La cláusula no limita sin embargo la información requerida a aquella que sea estrictamente necesaria para cursar la transacción. Por el contrario, la segunda autorización se refiere precisamente a la recopilación de información por el operador, que se extiende a la "derivada de los gustos, preferencias y en general de la utilización que hacen los Usuarios de los Servicios”. Pero lo cierto es que la cláusula ni siquiera limita a estos últimos parámetros la información que pueda requerir del consumidor. En tal sentido, la referencia a la información "proporcionada" por los usuarios es engañosa, pues es el propio operador del sitio quien determina qué información solicitar para procesar la transacción.

Al resolver el presente recurso esta Corte no ha sido llamada a calificar si esta ilimitada facultad para requerir información es o no contraria a la buena fe. Ella constituye sin embargo un antecedente de importancia para evaluar la conformidad de la autorización a revelar información que ahora se analiza: en la medida en que la información recopilada no aparece delimitada, la autorización a revelar información a terceros aparece asimismo ilimitada.

Por su parte, el inciso primero artículo 4 de la ley 19.628 sobre protección de datos de carácter personal dispone: "El tratamiento de los datos personales sólo puede efectuarse cuando esta ley u otras disposiciones legales lo autoricen o el titular consienta expresamente en ello”. La letra o) del artículo 2 de la misma ley, al definir lo que se entiende por tratamiento de datos, comprende "cualquier operación o complejo de operaciones o procedimientos técnicos, de carácter automatizado o no, que permitan... comunicar, ceder, transferir... datos de carácter personal”.

En consecuencia, la revelación de datos personales a terceros, salvo que lo autorice la ley o que el titular de los datos consienta en ello, es ilegal. El derecho protegido por estas disposiciones es la protección de la vida privada, explícitamente garantizado por el artículo $19 \mathrm{~N}^{\circ} 4$ de la Constitución Política. Resulta contraria a la buena fe, y en consecuencia abusiva, la obtención del consentimiento del titular de los datos mediante una condición general de contratación incluida en una transacción cuyo objeto principal es la entrada a un espectáculo. En el contexto de semejante transacción comercial, tal renuncia a la privacidad de los datos personales solo es válida si es otorgada en forma explícita y específica.

Duodécimo: Que la cláusula "Política de Privacidad de Ticketmaster", al autorizar a recolectar "información que es derivada de los gustos, preferencias y en general de la utilización que hacen los Usuarios de los Servicios”, también contraviene la buena fe en los términos proscritos por el artículo $16 \mathrm{~g}$ ) de la ley de protección de los derechos del consumidor. Dicha recopilación está también incluida en el concepto de "tratamiento de datos" que utiliza el inciso primero artículo 4 de la ley 19.628. En efecto, la ya citada letra o) del artículo 2 de dicha ley, define el tratamiento de datos como "cualquier operación o complejo de operaciones o procedimientos técnicos, de carácter automatizado o no, que permitan recolectar, almacenar, grabar... datos de 
carácter personal". Se trata por otra parte de información que excede de la necesaria para concluir las transacciones de compraventa de entradas, de manera que su recolección requiere o autorización legal o expreso consentimiento del titular de los datos. Por razones idénticas a las señaladas en el considerando precedente, resulta abusiva y nula esta cláusula en cuanto por ella se busca obtener tal consentimiento en forma atada a una operación comercial con un objeto diferenciado.

Decimotercero: Que la cláusula "Política de privacidad de Ticketmaster" también autoriza a la denunciada a utilizar la información obtenida a través del sitio "para diversos objetivos comerciales, como lo es el proporcionar datos estadísticos (por ejemplo: $50 \%$ de nuestros Usuarios son mujeres) a anunciantes potenciales, enviar publicidad a los Usuarios de acuerdo a sus intereses específicos, conducir investigaciones de mercadeo, y otras actividades o promociones que Ticektmaster considere apropiadas".

Esta cláusula contraviene lo dispuesto en el inciso segundo del artículo 3 de la citada ley de protección de la vida privada: "El titular puede oponerse a la utilización de sus datos personales con fines de publicidad, investigación de mercado o encuestas de opinión”. Esta prohibición no es más que una particularización de la prohibición genérica del artículo 4 de la misma ley citado en los motivos precedentes, pues tal utilización de datos personales también se encuentra incluida en la definición del "tratamiento de datos".

Por esta razón, también en este punto la cláusula "Política de privacidad de Ticketmaster" resulta abusiva y nula.

Decimocuarto: Que, por último, dicha cláusula autoriza a la denunciada a revelar la información "cuando por mandato de ley y/o de autoridad competente le fuere requerido o por considerar de buena fe que dicha revelación es necesaria para: I) cumplir con procesos legales; II) cumplir con el Convenio del Usuario; III) responder reclamaciones que involucren cualquier Contenido que menoscabe derechos de terceros o; IV) proteger los derechos, la propiedad, o la seguridad de Ticketmaster, sus Usuarios y el público en general". En rigor, todas estas son hipótesis en las cuales la revelación de información aparece en principio justificada, o al menos excusada, en el cumplimiento de la ley o mandato de autoridad, o en la protección de derechos propios o ajenos. En tales hipótesis, la revelación podría hacerse aun sin la autorización del titular de los datos. La cláusula cumple más bien una función de información al usuario de las limitaciones a las que la privacidad de sus datos queda sujeta en cumplimiento de la ley o de los derechos de los demás. En consecuencia, en este punto la cláusula no resulta abusiva. Esto es sin perjuicio de los abusos que puedan cometerse al amparo putativo de la cláusula, los que podrán dar lugar a las responsabilidades que establezca la ley.

Decimoquinto: Que es útil señalar que la sentencia impugnada omitió toda consideración para justificar el rechazo de la demanda en relación con la cláusula "Política de privacidad de Ticketmaster"; no obstante, con las consideraciones que se consignan en los motivos undécimo a decimotercero precedentes, queda demostrado que dicha sentencia incurrió en infracción de ley al desestimar que la cláusula "Política de Privadad 
de Ticketmaster", salvo en la parte a que se refiere en considerando anterior, y por ello infringe, por falta de aplicación, el artículo 16 letra g) de la ley 19.496 y, en consecuencia, se acogerá el recurso de casación en el fondo.

Decimosexto: Que el segundo capítulo del recurso de casación en el fondo denuncia la infracción del artículo 17 de la ley de protección de los derechos del consumidor al estimar que no se habría acreditado en el proceso el tamaño de la letra del boleto de entrada.

Decimoséptimo: Que el recurso no ha reclamado infringidas las leyes reguladoras de la prueba, de manera que esta Corte está forzada a aceptar los hechos que se han dado por probados por los jueces de instancia. No estando acreditado que las condiciones en los boletos de las entradas hayan tenido un tamaño inferior a los 2,5 milímetros, no resulta justificado que se haya incurrido en infracción del citado artículo 17.

A mayor abundamiento, cualquier infracción al artículo 17 carecería de influencia en lo dispositivo del fallo. En efecto, la cláusula incluida en los boletos de entrada fue, en todo caso, declarada abusiva. Y, según se ha consignado en el motivo cuarto precedente, la multa por infracciones a la citada genérica fue impuesta en su máximo legal.

Decimoctavo: Que, finalmente, el recurso reclama infracción a los artículos $51 \mathrm{~N}^{\circ} 2$ y 53 letras c) y d) de la ley de protección a los derechos del consumidor. Alega que la sentencia impugnada habría incurrido en dicha infracción al desestimar la petición de indemnización a los consumidores afectados.

Decimonoveno: Que la sentencia recurrida no dio por establecido hecho alguno que permita determinar si se incurrió en la infracción denunciada. En consecuencia, este capítulo del recurso será desestimado por exceder el ámbito propio de revisión del recurso de casación en el fondo.

Por estas consideraciones y de conformidad, además, con lo dispuesto por los artículos 764, 765, 766, 767, 768 y 785 del Código de Procedimiento Civil, se rechaza el recurso de casación en la forma deducido en contra de la sentencia de once de noviembre de dos mil catorce, escrita de fojas 440 y siguientes y se acoge el recurso de casación en el fondo en contra de la misma, la que se anula, dictándose a continuación y sin nueva vista, sentencia de reemplazo.

Regístrese.

Redactado por el abogado integrante señor Correa.

Rol No 1533-15

Pronunciado por la Segunda Sala integrada por los Ministros Sres. Haroldo Brito C., Carlos Cerda F., Jorge Dahm O., y los Abogados Integrantes Sra. Leonor Etcheberry C., y Sr. Rodrigo Correa G. No firma el Ministro Sr. Cerda, no obstante haber estado en la vista de la causa y acuerdo del fallo, por estar en comisión de servicios.

Autorizada por el Ministro de Fe de esta Corte Suprema.

En Santiago, a siete de julio de dos mil dieciséis, notifiqué en Secretaría por el Estado Diario la resolución precedente. 
Santiago, siete de julio de dos mil dieciséis.

En cumplimiento a lo dispuesto en el artículo 785 del Código de Procedimiento Civil, se dicta la sentencia de reemplazo que sigue.

Vistos:

Se reproducen los considerandos de la sentencia anulada de once de noviembre de dos mil catorce.

Y considerando:

Lo consignado en los considerandos octavo y undécimo a decimocuarto de la sentencia que antecede.

Se revoca la sentencia veinticuatro de marzo de dos mil catorce, que rola a fojas 326 de estos autos, en los siguientes puntos:

I. En cuanto por ella no se multó a la denunciada y, en su lugar, se declara que se multa a Ticket Master de Chile S.A. en la cantidad de 50 (cincuenta) Unidades Tributarias Mensuales, y

II. En cuanto ella desechó la demanda de declarar abusiva y nula la cláusula "Política de Privacidad de Ticketmaster" y, en su lugar, se la acoge y se declara que dicha cláusula es abusiva y nula, salvo en la parte en que autoriza a la denunciada a revelar información por mandato de ley o autoridad competente, o en protección de los derechos propios o de tercero.

Se confirma en lo demás la referida sentencia.

Regístrese y devuélvase, con su agregado.

Redacción del abogado integrante señor Correa.

Rol No $1533-15$.

Pronunciado por la Segunda Sala integrada por los Ministros Sres. Haroldo Brito C., Carlos Cerda F., Jorge Dahm O., y los Abogados Integrantes Sra. Leonor Etcheberry C., y Sr. Rodrigo Correa G. No firma el Ministro Sr. Cerda, no obstante haber estado en la vista de la causa y acuerdo del fallo, por estar en comisión de servicios.

\section{COMENTARio}

El caso "Sernac con Ticketmaster" comienza el 3 de enero de 2009 con la acción en defensa del interés difuso de los consumidores que interpone Sernac. En la demanda se alegaron diferentes infracciones a la Ley $\mathrm{N}^{\circ} 19.496^{1}$ (LPDC), a saber, la falta de información veraz y oportuna (art.3 b), vulneración del derecho a la libre elección (art.3 a), incumplimiento contractual (art.12), no informar precios de manera completa (art.30) y cláusulas abusivas (art. 16 letras c, d, y g). La alegación de nulidad por abusividad se solicitó respecto de las cláusulas contenidas en la página web de la demandada y en los tickets de las entradas a espectáculos o eventos en cuya comercialización Ticketmaster participó.

\footnotetext{
${ }^{1}$ Ley $\mathrm{N}^{\mathrm{o}} 19.496(07 / 03 / 1997)$, acerca de protección de los derechos de los consumidores.
} 
Este comentario se refiere, en específico, a una cláusula que figuraba en el sitio web y que en este fallo ha sido declarada abusiva por contravenir la buena fe en los términos del artículo $16 \mathrm{~g}$ ). Se trata de la cláusula denominada "Política de privacidad de Ticketmaster".

Tal como se lee en los considerandos undécimo y duodécimo, se trataba de una de las condiciones aplicables a la venta de entradas para espectáculos mediante el sitio web de la empresa demandada. Se estimó contraria a la buena fe, y en consecuencia abusiva, porque permitía revelar la información proporcionada por los usuarios a terceros, obteniendo el consentimiento para ello mediante esta condición general de la contratación que se entiende incluida en una transacción cuyo objeto principal es la entrada a un espectáculo, en circunstancias que tal renuncia a la privacidad de los datos personales solo es válida si es otorgada en forma explícita y específica.

Como señalan dichos considerandos, en este fallo se declara nula por abusiva una condición general de la contratación. Estas han sido definidas por De Castro como "un conjunto de reglas que un particular (empresario, grupo o rama de industriales o comerciantes) ha establecido para fijar el contenido (derechos y obligaciones) de los contratos que sobre un determinado tipo de prestaciones se propone celebrar" ${ }^{2}$. Así, la cláusula "Política de privacidad de Ticketmaster" contenía estipulaciones que estaban destinadas a ser parte integrante de los contratos de compraventa on line que se celebren sobre la entrada a alguno de los espectáculos en cuya comercialización la empresa demandada participaba. Lo anterior quiere decir que el control judicial de cláusulas abusivas, esta vez, no recayó en un contrato de adhesión, sino -como se viene diciendo- en una cláusula general de la contratación (CGC).

Como se desprende de la definición transcrita, uno de los elementos esenciales de las CGC es la preformulación ${ }^{3}$, lo que significa que se trata de cláusulas que cronológicamente preceden a la celebración del contrato porque justamente han sido expedidas con miras a su utilización en futuros contratos. Esto resulta relevante porque de acuerdo con la distinción entre control preventivo y control represivo de cláusulas abusivas ${ }^{4}$, el primero es aquel que opera antes de que el contrato haya sido introducido al mercado y el segundo tiene lugar cuando "los contratos ya se encuentran regulando la relación entre consumidores y proveedores" . Entonces, es posible concluir que el control que la Corte Suprema ejerció, en este caso, fue un control preventivo.

2 De Castro, F. "Las condiciones generales de los contratos y la eficacia de las leyes", en Anuario de Derecho Civil, tomo XIV, fascículo II, Madrid, 1961, 297 p.

${ }^{3}$ Es decir que "temporal, cronológicamente, están ya expeditas con anterioridad a la inclusión de las partes con miras a su utilización en futuros contratos”. Rezzonico, J. Contratos con cláusulas predispuestas. Condiciones negociales generales, Astrea, Buenos Aires, 1987, 123 p.

${ }^{4}$ García, M. Condiciones generales de los contratos, Editorial Revista de Derecho Privado, Madrid, 1969, pp.176 y ss.

${ }^{5}$ De la Maza , Í. "El control de las cláusulas abusivas y la letra G)”, en: Barrientos, F., De la Maza, I., y Pizarro, C. (Eds.), Consumidores, LegalPublishing Chile, Santiago, 2012, 116 p. 
El problema con esto es que no queda tan claro que en Chile los tribunales de justicia estén facultados para declarar la nulidad de CGC ejerciendo, de esta forma, control preventivo. En efecto, según el artículo 16 de la LPDC, al juez le compete ejercer control sobre "contratos de adhesión" 6 y no obstante la relación que existe entre una CGC y el contrato de adhesión, conceptualmente no son lo mismo. Las CGC son virtualmente contractuales y solo adquieren el valor de cláusula contractual cuando integran el todo o una parte de un contrato que, generalmente, será un contrato de adhesión. Es por eso que algunos las entienden como "una práctica comercial para imponer una oferta completa" 7 . Distinto habría sido si la acción de Sernac se hubiese ejercido en defensa del interés colectivo de los consumidores por la celebración de contratos de adhesión donde estas cláusulas se entiendan incorporadas porque allí hay control represivo y la situación cabe, sin dudas, dentro del marco legal ${ }^{8}$.

Por tanto, según el tenor del artículo 16 de la LPDC, el juez tiene conferida claramente la facultad para declarar nula una cláusula inserta en un contrato de adhesión ejerciendo, entonces, control judicial represivo. Sin embargo es factible preguntarse si es que puede el juez extender dicha facultad a CGC ejerciendo, sobre ellas, control judicial preventivo de cláusulas abusivas. Una respuesta afirmativa podría encontrar fundamento en el principio de protección de los consumidores, entendiendo que el juez interpretando ampliamente la norma puede extender su ámbito de aplicación para favorecer la protección de los derechos del sujeto débil. Una respuesta negativa podría encontrar sustento en el principio de juridicidad. El caso Ticketmaster habría sido el escenario ideal para debatir el asunto. Lamentablemente la sentencia no ofrece un análisis al respecto, y tampoco es posible encontrarlo en las sentencias de primera o segunda instancia.

Con todo, el máximo tribunal ha hablado, y con la declaración de nulidad de la CGC, denominada "Política de privacidad de Ticketmaster", ha ejercido control judicial preventivo de cláusulas abusivas.

${ }^{6}$ Artículo 16.- "No producirán efecto alguno en los contratos de adhesión las cláusulas o estipulaciones que:" pp. 25-26.

7 Tapia, M y Valdivia, J. Contrato por adhesión. Ley 19.496, Editorial Jurídica de Chile, Santiago, 1999,

${ }^{8}$ Control represivo propiamente tal. Un ejemplo es el caso Sernac con Cencosud. Corte Suprema, 24 de abril de 2013, Rol 12.355-11. 